\title{
La diversidad en los antropónimos de los peruanos
}

\section{Diversity in the anthroponyms of peruvians}

Marcelino Rojas Salazar ${ }^{1}$

Universidad SISE. Lima, Perú

marcelo17comunicacion@gmail.com

Citar como: Rojas, M. (2018). La diversidad en los antropónimos de los peruanos. Desde el Sur, vol. 10, nro. 2, pp. 331-345.

\section{RESUMEN}

El Perú tiene una población de 31237000 habitantes, según los resultados del último Censo Nacional ejecutado por el Instituto Nacional de Estadística e Informática (INEI) en 2017 . El $49,2 \%$ son hombres y el $50,8 \%$ son mujeres. El territorio peruano posee una riqueza cultural tanto en la arquitectura, la escultura, la pintura, la música, la danza, la gastronomía, la literatura y la lingüística. Este último aspecto se refiere a la diversidad de lenguas que se consideran idiomas en el país. Así, cada lengua posee su propia estructura fonológica, morfológica y sintáctica, además, de su propio sistema de antroponimia. Desde los últimos 25 años, los peruanos inscriben los nombres y apellidos de sus hijos mediante el organismo público llamado Registro Nacional de Identificación y Estado Civil (Reniec), que se encarga de la identificación y entrega del documento nacional de identidad (DNI). Por otra parte, ocurre una situación en la cual los padres registran a los futuros ciudadanos del país con nombres motivados por la moda e influenciados por la televisión, la música, un evento cívico, la política, el deporte o el cine; o sea, se van dejando de lado los nombres de los abuelos, de los tíos, del santo y patrón del pueblo o de los mismos padres como fuente de inspiración para registrar a sus hijos. Sin embargo, esta libre elección por nombres supeditados a la moda puede

1 Docente de la Facultad de Educación y de Estudios Generales de la Universidad Nacional Mayor de San Marcos, del Área de Humanidades de la Universidad Científica del Sur y del Departamento de Humanidades de la Universidad Privada del Norte. 
traer consecuencias en el proceso de desarrollo de la autoestima del niño, debido a que existen nombres que poseen una carga semántica belicosa, agresiva e iracunda en la sociedad. Por ello, los padres deben considerar esta situación para evitar futuros conflictos emocionales.

\section{PALABRAS CLAVE}

Antroponimia, nombres propios, cultura, peruanos, Reniec, nombres extravagantes

\section{ABSTRACT}

Peru has a population of 31 million 237 thousand inhabitants according to the last National Census by the Instituto nacional de estadística e informática -National institute for estatistics and informatics- (INEI) in 2017. 49.2\% are men and $50,8 \%$ are women. Peruvian territory is rich in architecture, sculpture, painting, music, dance, gastronomy, literature and linguistics. This last aspect refers to the diversity of languages in the country. Each language has its own phonological, morphological and syntactic structure, as well as its own anthroponymy system. Thus, in the last 25 years, Peruvians register names and surnames of their children through the public body called the Registro nacional de identificación y estado civil -National Registry of Identification and Civil Status- (Reniec), responsible for the identification and delivery of the Documento nacional de identidad -National identity document, (DNI). It happens that parents are naming future citizens with names motivated by fashion and influence by television, music, civic events, politicians, sportsmen or women and cinema actors, leaving aside names of grandparents, uncles, local town saint and patron or even parents as a source naming their children. However, this free choice by names linked to fashion can have consequences in the development process of the child's self-esteem, because sometimes they are names with warlike, aggressive and angry semantic charge in society. Therefore, parents should consider this situation to avoid future emotional conflicts.

\section{KEYWORDS}

Anthroponymy, own names, culture, Peruvians, Reniec, extravagant names 
La globalización es la nueva tendencia hacia el progreso en aspectos tan diversos como la economía o la tecnología. Las personas conviven con artefactos creados para su comodidad y el disfrute, y están influenciadas por las acciones y acontecimientos que ocurren en el mundo. Los personajes que se presentan como héroes o revolucionarios de una nueva vida están marcando la tendencia para emularlos, ya sea vistiendo o adoptando actitudes propias de ellos. Esta situación también influye en el sistema antroponímico, es decir, afecta al nombre que posee cada persona desde que su padre o apoderado lo inscribe legalmente en la institución encargada para esa función. Cada año, los padres peruanos designan a sus hijos con nombres de personajes populares mediáticos que se encuentran en la palestra de la farándula o del espectáculo. Poco a poco se va perdiendo la costumbre de nombrar a los hijos con el nombre de los abuelos, de algún familiar fallecido, de los mismos padres o de algún santo representante de la comunidad. Ahora la tendencia es elegir un nombre popular, y se cae así en el fanatismo y la poca responsabilidad en el desarrollo actitudinal del niño, porque los nombres están sujetos semánticamente a las acciones que cometen esos personajes de ficción. Es decir, si un actor o cantante comete actos negativos, delictivos o impropios ante la moral de la sociedad, el niño que posea su nombre estará inmerso en un conflicto emocional, ya que puede generarse el bullying en su escuela y arrastrar esta situación hasta su adultez. Además, el nombre puede tener una carga semántica favorable que resalta los valores morales, las buenas prácticas, el reconocimiento o, todo lo contario, puede tener una carga semántica perjudicial que enfatiza las acciones negativas, los antivalores, la criminalidad, el terrorismo y la degradación social. Por lo tanto, los padres deben ser conscientes y responsables al momento de elegir los nombres, ya que estos formarán parte de la vida legal y social de sus hijos.

A continuación, se presentará la disciplina lingüística que se encarga de estudiar los nombres, la institución que se encarga de registrar estos nombres en el país, los principales motivos que son la nueva tendencia antroponímica y las consecuencias negativas que los nombres excéntricos influyen en los futuros ciudadanos peruanos.

Los estudios sobre la antroponimia como una disciplina lingüística han logrado su sistematicidad metodológica como ciencia a partir de la década de 1940. En nuestro país, el antecedente más destacado es el cuestionario del Atlas etnográfico del Perú de 1980 (Huamán, 2002, p. 51), como una orientación para guiar los diversos trabajos de campo que se pueden elaborar en el rango de la antroponimia. Por un lado, también hay que considerar a la onomástica dentro de los estudios antroponómicos, 
ya que es parte de ella. De esa manera, Solís (1997, citado por Jacinto, 2009, p. 36) expresa lo siguiente:

La onomástica es la disciplina que estudia los nombres [propios], sean estos de personas o de lugares. En esta perspectiva, la onomástica tiene dos ramas principales: la antroponimia y la toponimia. La primera trata de los nombres de personas, mientras que la segunda, de los nombres de lugares. A su vez, tanto la antroponimia y más aún la toponimia pueden presentar subramas, dependiendo de una serie de consideraciones.

Por otro lado, Dubois (1986) menciona que la antroponimia forma parte de la onomástica y se encarga de estudiar la etimología e historia de los nombres propios de persona. De acuerdo con la Real Academia Española (2017), la antroponimia es en su primera acepción el «estudio del origen y significación de los nombres propios de persona» y en su segunda acepción es «el conjunto de nombres propios de persona». En cuanto a la definición de un nombre propio, Solís (1997, citado por Jacinto, 2009, p. 36) menciona: «De acuerdo [con] las tradiciones específicas, son los nombres de pila, los apellidos (patronímicos: padres o matronímicos: madres) así como los sobrenombres (apodos o alias) y los nombres diminutivos».

Cada nombre propio seleccionado por los padres para designar a sus hijos es determinado por la cultura donde reside o convive y por la época en que se desenvuelve. El sociólogo Edward Butnett (1929, citado por Jacinto, 2009, p. 35) propone que «la cultura es aquel conjunto complejo que comprende el conocimiento, las creencias, el arte, la moral, el derecho, las costumbres y cualquier otra capacidad y hábito adquirido por el hombre en cuanto miembro de una sociedad». Igualmente, Solís (2002, p. 224) afirma que «son normas y valores que funcionan como pautas de conducta de las personas o de grupos humanos. Los modos de ser, sentir y pensar compartidos. No solo es el patrimonio adquirido o heredado, es de por sí una entidad creativa y activa».

Es así como los peruanos encuentran una motivación al nombrar a los bebés cuando nacen, ya sea con sus propias creencias o razones, con sus propias normas morales y hábitos para la identificación. La investigadora Cuba (2002, p. 124, citando a Ullmann, 1972) declara que «Los nombres propios sirven de señales o marcas para que unos individuos puedan identificar a otros; en cambio, los nombres comunes son en sí mismos unidades significativas». Se puede afirmar entonces que las personas poseen un patrón de nombramiento de manera regular dependiendo la época y de sus expectativas, como lo menciona Unicef-CILA: «La denominación - poner nombres - es un proceso sistemático en las culturas. Se actualiza cada vez que se nomina. Toda cultura tiene subsistemas de 
denominación: uno sumamente dinámico y productivo es aquel que asigna nombres a los seres humanos, cuyo alcance es de carácter universal» (2012, p. 26).

Los antropónimos poseen dos rasgos propios de su naturaleza: uno clasificador y otro simbólico. Cada uno de ellos otorga la capacidad de transmitir algunos valores culturales, étnicos y poéticos. El lingüista Jacinto define ambos rasgos de la siguiente manera:

En su calidad de clasificadores, los nombres indican pertenencia de un individuo a un grupo social $y$, a veces, profesional, el origen geográfico, el rango o estatuto de nacimiento, y aportan una información de identidad sexual y hasta generacional, referida a las franjas de edad. Como símbolos, representan un sistema de valores y creencias (nombres teóforos, míticos...), participando de una determinada visión del mundo (2009, p. 38).

En nuestro país, una persona suele identificarse por tres o más antropónimos: nombre de pila (referente a la pila bautismal, ya que el nombre que se da a una persona cuando nace y con el que se inscribe en el registro civil, puede ser confirmado con el bautismo), apellido paterno y apellido materno. Ahora bien, la selección de los nombres de pila puede estar motivada por una tendencia hacia lo popular y mediático, es decir, pueden existir razones que evocan a situaciones de felicidad donde se ha vivido un momento agradable y se desea perennizar de una forma legal ante la comunidad. «La elección de los nombres obedece a distintas razones, que van desde la originalidad, la evocación, la estética, el esnobismo o la moda, a factores étnicos o a motivos religiosos, que pueden responder a la tradición y a las normas sociales» (Jacinto, 2009, p. 42).

El derecho a la identidad y al nombre de las personas está respaldado por la Constitución Política del Perú de 1993, en el título I, capítulo 1, artículo 2 e inciso 1, que dice: «Toda persona tiene derecho a la vida, a su identidad, a su integridad moral, psíquica y física y a su libre desarrollo y bienestar». De igual modo, en el artículo 6 del Código de los Niños y Adolescentes: «tienen derecho a un nombre, a la nacionalidad peruana, a conocer a sus padres y a ser cuidado por estos. Será registrado por su madre o responsable inmediatamente después de su nacimiento en el registro civil correspondiente».

Como bien se menciona, los bebés son inscritos en el Registro Nacional de Identificación y Estado Civil (Reniec) por el declarante, que puede ser la madre, el padre o ambos, dentro de los 60 o 90 días de nacido. Este organismo público y autónomo fue creado por la Constitución Política y se establece como un registro de seguridad jurídica cuya finalidad es la identificación de las personas naturales otorgando el documento 
nacional de identidad y registrando hechos vitales como nacimientos, defunciones, divorcios y otros que modifican el estado civil. Hay que precisar que, antes de la creación del Reniec, todas esas actividades se realizaban en las municipalidades o en las parroquias de los pueblos alejados. Todos los libros y las actas registradas se encontraban en sus almacenes.

Hace 30 años no existía un recuento ni contabilidad de los nombres más comunes del Perú. Ahora, con la tecnología y los programas informáticos especializados en el registro de personas, se puede conocer esta diversidad nominal, desde el nombre más utilizado hasta el menos común. Por ello, desde hace cuatro años el Reniec publica en su página web institucional y en su Facebook oficial la diversidad de nombres que se inscriben anualmente. Desde los nombres y los apellidos más utilizados hasta los menos usuales y poco ortodoxos.

En julio de 2018, Reniec hizo la entrega de la lista actualizada de los 40 nombres más utilizados por los peruanos. Entre los nombres para los varones se encuentran: José (696 021 personas), Luis (556 570), Juan (554 667), Carlos (369 754), Jorge (274 967), Víctor (237 940), César (193 858), Miguel (190 599), Jesús (186 034), Julio (183 114), Pedro (156 907), Manuel (145 890), Santos (128 197), Jhon (124 212), Ángel (123 744), David (119 412), Segundo (119 082), Diego (117 990), Daniel (110 190), Óscar (100 954), Marco (96 985), Edwin (94 494), Javier (91 415), Walter (89 846), Fernando (88 666), Alejandro (86 778) y Álex (84 199 personas). Entre los nombres para las mujeres están los siguientes: María (928 240 personas), Rosa (308 561), Ana (215 099), Luz (207 411), Carmen (193 865), Juana (142 066), Flor (112 718), Julia (108 713), Elizabeth (106 041), Ruth (104 167), Diana (100 011) y Milagros (88 903 personas).

Por otro lado, también se han considerado los 40 apellidos con mayor recurrencia en el país. Estos son: Quispe (1 212114 personas), Flores (763 245), Sánchez (680 395), García (658 376), Rodríguez (628 844), Huamán (610 076), Rojas (606 030), Vásquez (551 670), Mamani (524 993), López (524 818), Ramos (515 074), Pérez (504 766), Torres (497 217), Díaz (484 625), Gonzales (467 855), Ramírez (463 411), Mendoza (436 960), Chávez (431 591), Espinoza (396 087), Castillo (372 605), Fernández (370 919), Vargas (344 833), Gutiérrez (343 026), Cruz (340 716), Ruiz (328 722), De la Cruz (287 861), Romero (270 278), Gómez (269 305), Silva (264 121), Córdova (257 639), Condori (256 114), Castro (255 639), Martínez (244 654), Reyes (244 591), Rivera (240 375), Salazar (232 394), Medina (229 899), Aguilar (229 592), Morales (225 006) y Paredes (224 520 personas).

El presente estudio se focalizará únicamente en los nombres de las personas — «nombre de pila», cuya expresión ya se explicó en un párrafo anterior-y las diversas influencias en la decisión de elegir los nombres 
para los niños. Por lo general, estas personas seleccionan el nombre o los nombres por diversas razones: los nombres de los abuelos, los nombres de los tíos, los mismos nombres de los padres, los nombres de las ciudades, los nombres de actores o actrices populares. No obstante, en los últimos años, los peruanos han elegido con mayor regularidad la fama de personajes del medio artístico, deportivo, musical o eventos de trascendencia nacional o internacional. Según esta visión, se explicará las razones de selección nominal y se describirá el listado de nombres con la cantidad de personas que poseen esta nominación.

La publicación de Reniec indica que, entre enero y marzo de 2015, los nombres preferidos por los peruanos al momento de registrar a sus hijos estuvieron motivados por los personajes protagonistas de una serie de televisión de procedencia turca llamada Las mily una noches: Onur (7 personas) y Sherezade (12 personas). En otro año, se dio a conocer los nombres más utilizados para el registro en los bebés nacidos durante 2017. Ellos fueron los siguientes: en los varones, Thiago (8295 personas), Liam (8112) y Dylan (4903); en las niñas, Mia — sin tilde - (3767) y Camila (3635 personas).

De igual forma, en mayo de 2015, se publicó que el Día de la Madre (celebrado el segundo domingo de mayo) se presenta como otra motivación, ya que existen mujeres que llevan la maternidad en el nombre: Madre (19 personas), Mami (3), Mamita (2), Madrecita (1), Mama — sin tilde- ( 2 personas). También en la lengua asháninca se presenta Ina (que significa "mami", 592 personas) y, en la lengua shipibo, Tita (que significa "mamá", 231 personas). Así también, en junio del mismo año, se publicó que otra motivación para elegir un nombre es el homenaje al progenitor por el Día del Padre (celebrado el tercer domingo de junio). Allí se encuentran: Papi (18 personas), Paterno (14), Papito (7), Padre (2), Guía (1 persona).

Cada 6 de julio se celebra el Día del Maestro. En esta fecha se le considera su reconocimiento por la vocación de servicio y la ilustre tarea de formar a los hombres y mujeres del país, y por transmitir el conocimiento más allá de lo académico y educar en valores. Por eso, este personaje inspira otra motivación para la selección de un nombre. Así se tiene: Maestro (1 persona), Profesora (1), Tarea (1). De igual forma, los nombres de personajes de televisión que cumplieron el rol de maestro: Ximena — de la telenovela mexicana Carrusel- (16 131 personas), Jacinta — de la serie de televisión infantil argentina Señorita Maestra- (8957), Miyagui —de la película estadounidense Karate Kid - (31 personas), Miyagi (12), Roshi —del anime japonés Dragon Ball- (9), Yoda — de la película Star Wars(4 personas). Por otro lado, se ha considerado a personajes ilustres de la 
historia peruana como el educador José Antonio Encinas (21 personas), José Carlos Mariátegui —el Amauta - (10), el escritor indigenista José María Arguedas (8), el historiador de la República Jorge Basadre (7) o el maestro y prócer independentista José Faustino Sánchez Carrión (4 personas).

En marzo de 2016, Reniec dio a conocer que por motivo del Día Internacional de la Mujer (celebrado el 8 de marzo de cada año) se presenta otra motivación con nombres alusivos al reconocimiento de las mujeres que cumplieron un rol significativo en la historia del Perú. De esa manera están: Rosa Merino - soprano y primera intérprete del himno nacional del Perú- (543 personas), Antonia Moreno - dirigió la resistencia en la sierra central contra Chile durante la Guerra del Pacífico- (123), Mercedes Cabello - escritora e iniciadora de la novela realista peruana- (53), Sarita Colonia - joven a quien se le rinde culto y veneración porque se le atribuye la capacidad de hacer milagros - (40), Clorinda Matto - escritora y precursora del género indigenista - (5) y Chabuca Granda - cantautora de valses criollos y ritmos afroperuanos- (4). Del mismo modo existen: Eva (35 142 personas), Bella (4810), Venus (877), Coya (95), Dama (13), Señora (6), Mujer (4), Warmi (3), Señorita (2), Doncella (2), Chica (1), Femenina (1), Niñita (1) y Compañera (1 persona).

Las personas siempre tienen una preferencia por algún artista, ya sea porque actúa muy bien o porque ha servido de inspiración en sus relaciones amorosas que las han llevado hacia el matrimonio. Es así como en agosto de 2016, Reniec manifestó - tras el fallecimiento del cantautor, actor, compositor, músico y productor discográfico mexicano Alberto Aguilera Valadez, más conocido como Juan Gabriel— que 5094 personas llevan su nombre.

Asimismo, debido a la popularidad y el incremento de fanáticos de alguna película o serie de televisión de gran aceptación, los peruanos han considerado usar los nombres, por ejemplo, de los personajes de la exitosa serie japonesa de anime Dragon Ball Z: Gohan (169 personas), Shen (114), Roshi (18: se incrementó a diferencia de 2015), Krilin (12), Jiren (4), Wiss (4), Goten (10), Gokú (2), Piccolo (4), Trunks (11), Bills (26), Vegeta (2) y Freezer (1 persona).

Otra razón de selección nominal se debe al éxito de la saga Star Wars desde 1977. El fanatismo hacia estas películas se refleja en los nombres de sus hijos: Leia (533 personas), Han (365), Orson (210), Luke (198), Anakin (104), Arturito (20), Andor (8), Kenobi (8), Cassian (4), Obi (4), Vader (2), Darth (1), Obi-Wan Kenobi (1), Skywalker (1 persona).

En julio de 2017, Reniec midió la popularidad de la serie de televisión estadounidense de fantasía medieval, drama y aventuras Game of 
Thrones (Juego de tronos) según el uso de los nombres de sus personajes en la población peruana. Así se tiene: Khalessi (2482 personas), Daenerys (327), Bran (224), Arya (217), Sandor (126), Joffrey (112), Ramsay (37), Tully (7), Robb (6), Snow (5), Sansa (2) y Tyrion (2 personas).

Por otro lado, en enero de 2018 , se publicó la relación de los nombres de los personajes de la serie ochentera de anime japonesa Supercampeones - conocida como Captain Tsubasa-, que también ha sido la motivación de los padres al elegir los nombres para los niños. Es así como existe: Benji (869 personas), Misaki (144), Tsubasa (10), Hyuga (3), Tom Misaki (2), Oliver Atom (2) y Aoi Shingo (1 persona).

Los peruanos se caracterizan por demostrar una actitud afectuosa y sentimental frente a los eventos de celebraciones o conmemoraciones referidas a fechas cívicas a nivel nacional o mundial. El cuarto domingo de julio es homenajeado el pisco, la bebida bandera y el ingrediente principal en muchos cocteles peruanos, que también ha inspirado nombres en la población: Pisco (1 persona), Mosto (2), Negra (3), Uvina (8), Italia (249) y Albila (5 personas).

En febrero de 2017, Reniec informó que los peruanos se inspiran en el sentimiento más profundo del ser humano para inscribir los nombres en sus hijos. Cada 14 de febrero se celebra el Día de San Valentín, en el que se manifiesta el amor y la amistad entre las personas, y cada 13 de abril se celebra el Día Internacional del Beso. Ambos eventos motivan e influyen en la decisión de nombrar a sus hijos: Amador (11 677 personas), Valentín (697), Felicidad (598), Feliz (327), Amor (319), Caricia (230), Afrodita (165), Idilio (155), Tesoro (95), Love (68), Leal (42), Cariño (37), Corazón (29), Beso (23), Amoroso (21), Amante (17), Kiss (14), Ternura (13), Amore (12), Casanova (12), Amistad (10), Mi cielo (10), Pasión (9), Married (6), Friendly (5), Sincero (4), Celos (3), Cupido (2), Confianza (2), Amigo (2), Querer (1), Mi amor (1), Esposo (1), Prometido (1), Te Quiero (1), Cariñoso (1), Besito (1) y Amorcito (1 persona).

Según el último censo del Instituto Nacional de Estadística e Informática (INEI), el 76\% de la población peruana practica la religión católica. En julio de 2017, Reniec hizo un listado de nombres alusivos a la Semana Santa y a los personajes de la Santa Biblia: Maria — sin tilde - (1 251150 personas), Pedro (192 905), Cruz (24 000), Santa (19 826), José (18 783) y María Magdalena (18 666), Jesús (17 881), Dios (9849), Dimas (4839), Mesías (4098), Fe (2311), Nazareth (1665), Nazaret (799), Nazareno (599), Cristo (402), Betania (323), Evangelio (169), Simón (142), Judas (53), Herodes (31), Inri (27), Jerusalén (20), Jesucristo (12), Barrabás (3) y Resurrección (3 personas). 
En diciembre de 2017, Reniec informó que la Navidad no solo es un fuente de celebración llena de amor, ternura y tradiciones, sino también la razón por la cual se plasman los nombres relacionados con los personajes de este fecha: Maria — sin tilde- (1 271228 personas: se incrementó en cinco meses), Jose — sin tilde- (846 863), Jesus — sin tilde- (410 884), Ángel (331 536), Gabriel (131 779), Ángeles (36 168), Natividad (36 519), Estrella (29 701), José (14 652), Jesús (14 035: disminuyó en cinco meses), María (7420), Noel (8103), Baltazar (7349), Belen —sin tilde- (41 805), Pastor (6874), Paz (4970), Melchor (5028), Gaspar (4645), Belén (4310), Navidad (195), Niño (307), Noela (76), Reno (52), Guirnalda (17), Nacimiento (6), Trineo (4), Pastorcita (3) y Regalo (3 personas).

De igual modo, la fiesta navideña ha influido en las lenguas andinas como el quechua con los nombres: Wilin (que significa "Belén", 181 personas), Marya (que significa "María”, 116), Quyllur (que significa "Estrella", 50), Anjil (que significa "Ángel", 5), Qullar (que significa "Estrella”, 5), Jushi (que significa "José", 3), Jishu (que significa "Jesús", 2) y Milchu (que significa "Melchor", 1). La lengua aimara con los nombres: Warawara (que significa "Estrella", 2 personas) y Yuriña (que significa "Nacimiento", 2 personas).

Asimismo, la actividad festiva donde se reconoce la buena ventura y el buen augurio durante el año próximo, es decir, la celebración por el Año Nuevo, también se considera otro motivo para registrar a los niños. Estos nombres son los siguientes: Cena (307 personas), Brindis (212), Uva (51), Nuevo (19), Próspero (10), Deseo (4), Amarillo (2), Prosperidad (2), Año (1), Ritual (1) y Feriado (1 persona).

Se dice que el trabajo dignifica al hombre y que los peruanos son personas creativas, luchadoras y laboriosas que vencen a la adversidad. Esta situación estará presente cuando cada 1 de mayo se celebre el Día del Trabajo y se recuerde los nombres referidos a oficios y profesiones, como los siguientes: Marino (19 922 personas), Estilista (66), Actriz (27) y Obrero (21 personas).

Un equipo de profesionales de social media y community management en Estados Unidos, dirigidos por Jeremiah Owyang, decidió elegir el cuarto lunes de enero de cada año, desde 2010 como el Día de Reconocimiento al Community Manager. El Perú no estuvo alejado de esta actividad que pretende asegurar el bienestar de empresas, marcas, servicios y productos. Reniec hizo público los nombres que hacen referencia a este reconocimiento: Banner (460 personas), Link (70), Roi (70), Email (27), Hosting (19), Share (10), Avatar (7), Dominio (5), Fans (4), Chat (3), Manager (2), News (2), Branding (2), Feed (1), Digital (1), Web (1 persona). 
En julio de 2017, también se publicó otro motivo por el cual las personas llevan el patriotismo inmerso en sus nombres y aluden al aniversario de la independencia peruana. Tanto es su amor hacia la patria que los padres demuestran ese cariño inscribiendo a sus hijos con los siguientes nombres: Perú (15 personas), Patria (17 personas), Peruano (1 persona), Peruana (3 persona) e Independencia (1 persona).

La comunidad de los zurdos peruanos estableció el Día Internacional del Zurdo. En agosto de 2017, Reniec informó sobre los nombres de los personajes zurdos que han sobresalido en el mundo: Albert Einstein (89 personas), Isaac Newton (34), Marilyn Monroe (3), Barack Obama (17), Diego Maradona (9), Justin Bieber (17), Messi (2553), Einstein (941), Hendrix (320), Maradona (282), Obama (62), Brad Pitt (12), Bob Dylan (7), Pelé (7), Bowie (5), Jimi Hendrix (4), Chaplin (2) y Bill Gates (2 personas).

El arte también ha sido otra fuente de inspiración de muchos padres para nombrar a sus hijos y así expresar un reconocimiento a la película, el actor, la actriz o el personaje que les evoca algún momento impactante en sus vidas. De esa forma, en julio de 2017, se conocieron los nombres que evocan al sétimo arte por sus personajes en películas de terror y fantásticas: Jason (3359 personas), Samara (2163), Alien (27), ET (10), Darth (1) y Chucky (1); personajes de caricaturas infantiles: Dominic (5313), Igor (1943), Blanca Nieves (723), Dorothy (517), Mickey (284), Dory (223), Gigi (223), Woody (65), Minnie (55), Thor (49), Moana (42), Hulk (21), Batman (20), Cenicienta (13), Clark Kent (13), Nemo (9), Rapunzel (7), Luisa Lane (6), Peter Parker (5), Frozen (4), Bruce Banner (3), Bruce Wayne (2), Sirenita (2), Ironman (1), Mulan (1), Spiderman (1), Pinocho (1), Pluto (1) y Tribilín (1); personajes de películas de acción, lucha y poder: Rocky (1152 personas), Leia (606), Maximus (57), Aragom (23), Matrix (14), Van Helsing (10), Cyrano (7), Sirius (6), Loki (2), Vader (2), La roca (1); personajes protagonistas de películas: Ben Hur (83), Rambo (49), Django (46), Juana de Arco (30), Robin Hood (23), Espartaco (21), James Bond (16), Superman (8), Mad Max (7), Pantaleón (6), Troya (6), Spock (5), Frankestein (4), William Wallace (4), Tony Montana (3), John Connor (2), Jurassic (2), Rocky Balboa (2), Titanic (2), Xmen (2), Zorro (2), Bridget Jones (1), Chucky (1), Harry Potter (1), Tony Stark (1), Madeinusa (1) y Grease (370 personas).

La música también ha influenciado en el pensamiento de la población, especialmente al momento de registrar a los futuros ciudadanos del Perú. En julio de 2017, Reniec difundió la relación de nombres que aluden a los famosos intérpretes del rock y pop: Rihanna (2891 personas), Prince (1256), Sting (549), Nirvana (547), Billy Joel (302), Elton John (111), Queen (99), Elvis Presley (67), John Lennon (49), Toto (46), Journey (45), Rush (37), Bryan Adams (35), Peter Gabriel (35), Blondie (31), Mick Jagger (31), George 
Harrison (20), Oasis (20), Bon Jovi (17), Kiss (15), Bono (13), James Taylor (13), Slayer (12), Aerosmith (8), Brian Wilson (8), Bob Dylan (7), Steven Tyler (6), Jimi Hendrix (4), Korn (4), Avril Lavigne (3), Axl Rose (3), Janis Joplin (3), Morrisey (3), Git (3), Jim Morrison (2), Libido (2), Rod Stewart (2), Amy Winehouse (1), Bjork (1), Bruce Springsteen (1), Dire Straits (1), La Sarita (1), Paul Mccartney (1), Pearl Jam (1), Phil Collins (1), Rolling Stones (1) y Van Halen (1 persona).

Por último, en el Mundial de Fútbol de la Copa FIFA Rusia 2018, donde el equipo peruano participó luego de 36 años de ausencia, se presentó un listado de nombres referidos a este acontecimiento y a los futbolistas que destacaron en el proceso de clasificación. Los nombres son los siguientes: Mundial (3 personas), Paolo Guerrero (5), Aquino (3), Polo (11), Yotún (11), Gallese (1), Guerrero (5), Advíncula (1), Rodríguez (2), Ramos (1) y Farfán (1). Y otras estrellas del fútbol extranjero como: Cristiano Ronaldo (870), Lionel Messi (232), Antoine Griezmann (23), Toni Kroos (4), Eden Hazard (3), Harry Kane (3), Neymar Da Silva (3), Robert Lewandowski (1) y Kevin de Bruyne (1 persona).

Como se ha podido observar y analizar, la mayoría de nombres ha tenido diversas motivaciones para su elección por parte del padre o la madre del niño. Así también, existen casos donde el nombre y el apellido de un artista se han transformado en una unidad léxica, consolidándose en un nombre de pila. Algunas veces se ha respetado las grafías de origen en los nombres extranjeros, en otros casos ha ocurrido una adaptación ortográfica e incluso hay nombres que solo se diferencia por la presencia de la tilde. Al final, son los padres quienes deciden inscribir el nombre de sus hijos. Así como lo explica Jacinto: «La teoría del control cultural se basa en la idea de que un pueblo o grupo étnico ejerce decisiones sobre sus elementos culturales como grupo social. De allí parte la relevancia de que el pueblo o grupo regula su funcionamiento y reproducción» (2002, p. 35). Aunque a veces esta decisión de elegir nombres o combinaciones de nombres y apellidos de un artista como un nombre de pila para el hijo es creada para evitar la homonimia, ya que en el 2014, según la base de datos del Reniec, publicó que se presentaron 913765 personas que tienen homónimos y en su futuro próximo puede causarles problemas legales de documentación así como la confusión con alguna persona requerida por la justicia peruana.

De igual manera, existen nombres que pueden causar algún conflicto emocional en la formación de la actitud y personalidad de los niños, porque los personajes que los inspiraron hacen referencia a la violencia, el caos, el terror o la muerte, o tienen problemas legales, sociales y psicológicos. Esta situación puede generar el bullying en la escuela o el maltrato 
en la comunidad; es decir, se produce la burla por tener un nombre que tal vez para el padre sea significativo, pero para el hijo sea una condena. Así también lo afirma Huerta (2017):

Dos estudios publicados en The Journal of Social Psychology exponen que tanto varones como mujeres que tienen nombres raros o excéntricos, la mayoría nombrados como sus artistas o personajes favoritos al estilo Shakespeare o James Bond, tienen mayor tendencia a padecer trastornos mentales que deforman el pensamiento racional (párr. 8).

En síntesis, se ha presentado la diversidad de nombres en la población peruana que son motivados por aspectos mediáticos y populares en los últimos años. El sistema antroponímico cada vez se sistematiza con esta tendencia nominal tanto en su estructura como su semántica. Si bien los padres tienen la potestad de elegir y nombrar a sus hijos con plena libertad, eso no implica que se le otorgue un nombre extraño ajeno a nuestra cultura y que perjudique emocionalmente al niño durante su crecimiento. De igual manera, antes de la inscripción por primera vez en Reniec, el funcionario público debería organizar campañas informativas para recomendar a los padres sobre las consecuencias desfavorables que se pueden generar en el futuro de sus hijos. Finalmente, todos los datos cuantitativos que se han presentado en los nombres son publicaciones desde la base informática del Reniec. Por lo tanto, las cantidades de nombres por los habitantes pueden variar según la época, debido a que existe un porcentaje mínimo de habitantes que aún no obtienen su documento de identidad hasta el momento y por ende no se encuentran registrados en el sistema.

\section{Contribución del autor}

Marcelino Rojas ha participado en la concepción del artículo, la recolección de datos, su redacción y aprobación de la versión final.

\section{Fuente de financiamiento}

Autofinanciado.

\section{Conflictos de interés}

El autor declara no tener conflictos de interés. 


\section{REFERENCIAS BIBLIOGRÁFICAS}

Congreso Constituyente Democrático (1993). Constitución Política del Perú. De la persona y de la sociedad. Recuperado de http://www.pcm. gob.pe/wp-content/uploads/2013/09/Constitucion-Pol\%C3\%ADtica-delPeru-1993.pdf

Cuba, M. (2002). Antroponimia e identidad de los negros esclavos en el Perú. Revista Escritura y Pensamiento, vol. 5, nro. 11, pp. 133-134. Recuperado de http://sisbib.unmsm.edu.pe/bibvirtual/publicaciones/escri_pensam/2002_n10/antroponiamia_identidad_negros.htm

Dubois, J. y otros (1986). Diccionario de lingüística. Madrid: Alianza.

ElComercio.pe (2015). Maestro, Profesora y Tarea, así se llaman algunos peruanos. Recuperado de http://archivo.elcomercio.pe/sociedad/lima/ maestro-profesora-y-tarea-asi-se-llaman-algunos-peruanos-noticia1823893?ref=flujo_tags_518998\&ft=nota_58\&e=titulo

Huerta, E. (2017). Así es cómo el nombre puede condicionar nuestra personalidad. Recuperado de http://vital.rpp.pe/cuerpo-y-alma/asi-es-como-nuestro-nombre-puede-condicionar-nuestra-personalidad-noticia-1070344

Huamán, A. (2003). El léxico taurino. Estudio semántico, lexicográfico y antroponímico (tesis de licenciatura en Lingüística). Universidad Nacional Mayor de San Marcos, Lima. Recuperado http:// cybertesis.unmsm.edu.pe/bitstream/handle/cybertesis/2944/Huaman_ ca.pdf?sequence=1\&isAllowed=y

Instituto Nacional de Estadística e Informática (2017). Población de Perú totalizó 31 millones 237 mil 385 personas al 2017. Recuperado de https:// www.inei.gob.pe/prensa/noticias/poblacion-del-peru-totalizo-31-millones-237-mil-385-personas-al-2017-10817/

Jacinto, P. (2009). Estudio del sistema de denominación antroponímica de la cultura asháninka. Tesis para optar el título profesional de Licenciado en Lingüística. Lima: Universidad Nacional Mayor de San Marcos. Recuperado de http://cybertesis.unmsm.edu.pe/handle/cybertesis/954

LaRepública.pe (2017). Reniec revela cuántos peruanos llevan nombres de futbolistas peruanos. Recuperado de: https://larepublica. pe/sociedad/1 108647-reniec-revela-cuantos-peruanos-Ilevan-nombres-de-futbolistas-peruanos

Ojo.pe (2017). Reniec comparte lista de nombres símbolos de Navidad. Recuperado de https://ojo.pe/ciudad/reniec-lista-nombres-simbolos-navidadpero-olvida-merry-christmas-250296/?ref=bl-correo

Real Academia Española (2017). Diccionario de la lengua española [versión electrónica]. Recuperado de http://dle.rae.es/ 
Registro Nacional de Identificación y Estado Civil (2014). Derecho a la identidad versus derecho al nombre. Recuperado de http://sisweb.reniec. gob.pe/PortalRegCivil/getFilePub.htm?hoja=245.pdf

https://www.facebook.com/RENIECPERU/

(2017). Reniec Perú. Sitio web oficial. Recuperado de

Rojas, M. (2017). La influencia del chat en la escritura de los estudiantes. Desde el Sur, nro. 9, vol. 2. Recuperado de http://revistas.cientifica.edu.pe/ index.php/desdeelsur/article/view/367

(2017). Estrategias cognitivas y los niveles de comprensión lectora en los estudiantes del VI ciclo del nivel secundaria de la Institución Educativa Nro. 6081 Manuel Scorza Torres de San Gabriel Alto en Villa María del Triunfo. Recuperado de http://cybertesis.unmsm.edu.pe/handle/cybertesis/7020

Solís, G. (1997). La gente pasa, los nombres quedan... Introducción en la toponimia. Lima: Ediciones Lengua y Sociedad.

Trome.pe (2018). ¡Que no te troleen! Reniec revela cuántos peruanos se Ilaman Web, Email, Chat, Link, Fans y Troll. Recuperado de https://trome. pe/actualidad/reniec-revela-peruanos-Ilaman-nombres-web-email-chatlink-fans-troll-fotos-73563

Unicef-CILA (2012). Asháninka. Territorio, historia y cosmovisión. Recuperado de https://www.unicef.org/peru/spanish/Ashaninka-territorio-historia-cosmovision-Educacion-intercultural-bilingue.pdf 